\title{
A new implementation of BEM by an expanding element interpolation method
}

\author{
Jianming Zhang*, Lei Han, Weicheng Lin, Yunqiao Dong, Chuanming Ju
}

\begin{abstract}
A new implementation of boundary element method (BEM) by an expanding element interpolation method is presented in this paper. The expanding element is achieved by collocating virtual nodes along the perimeter of the traditional discontinuous element. With the virtual nodes, both continuous and discontinuous fields on the domain boundary can be accurately approximated, and the interpolation accuracy increases by two orders compared with the original discontinuous element. The boundary integral equations are built up for the inner nodes of the traditional discontinuous elements, only (taking these nodes as source points), while the virtual nodes are used for connecting the shape functions at the source points, thus the size of the final system of linear equations will not increase. The expanding element inherits the advantages of both the continuous and discontinuous elements while overcomes their disadvantages. Successful numerical examples with different boundary conditions have demonstrated that our new implementation is very encouraging and promising.
\end{abstract}

Keywords: boundary element method (BEM); interpolation method; expanding element;

\section{Introduction}

The boundary element method (BEM) has been widely used for solving engineering and scientific problems [1-13]. Compared with the finite element method (FEM), the BEM is more attractive for its dimension reduction feature and higher accuracy. In addition, the trial functions in the FEM formulation must be at least $C^{0}$ - 
continuous, which is not required in the BEM. However, how to take full advantages of this point has been a long-standing issue in the BEM community, and so far has not achieved an agreement.

There are two basic ways of implementing the BEM. The first way is to use the discontinuous elements. In this implementation, the collocation nodes are located inside the elements and the solution obtained is discontinuous at the interfaces between elements. The discontinuous elements provide a lot of convenience for implementation, including the simplification in the assembly and solution of the system equations, reducing the difficulty of mesh generation and easy computation of the 'free' terms appearing in the integral equations. But the number of degrees of freedom increases rapidly and much more CPU time and memory capacity are required. The second way is using the continuous elements in which the collocation nodes coincide with the geometrical vertices of the element. With the continuous elements, the interelement continuity of field variables can be obtained. But it could be difficult for the continuous elements to deal with some situations for instance the corner problems [14-16]. In addition, the standard continuous elements cannot meet requirements of differentiability and continuity at the source point in the hypersingular boundary integral equation [17-18]. The continuous and discontinuous elements each have their own advantages and disadvantages, and have been controversial ever since a long time ago [19-20].

To avoid the contradiction between the continuous and discontinuous elements, a new expanding element is proposed in this paper. The expanding elements not only possess the advantages of discontinuous element but also guarantee the interelement continuity. Furthermore, the discontinuous fields on the boundary can also be accurately approximated.

The new element is achieved by collocating virtual nodes along the perimeter of the traditional discontinuous element. These virtual nodes are used for connecting the source points rather than acting as the source points. The computation of the values of the virtual nodes is classified into three cases. (i) When interpolating the known boundary variables, the values of the virtual nodes equal the corresponding boundary conditions. As a consequence, the boundary conditions are accurately imposed. (ii) 
While interpolating the unknown boundary variables, the values of the virtual nodes are determined by all adjacent elements including the virtual nodes. For example, the value of the virtual node between two constant elements equals the average value of these two elements. Thus the interelement continuity is guaranteed. (iii) For interpolating discontinuous boundary variables, two virtual nodes are collocated at a vertex shared by two adjacent elements, one virtual node for one element, respectively. The values of these two virtual nodes are determined by their corresponding element. Using this scheme, the discontinuity of the boundary variables can also be accurately captured. In all these cases, the virtual nodes improve the interpolation accuracy; meanwhile the size of the final system of linear equations does not increase.

This paper is organized as follows. Section 2 introduces the expanding element interpolation method. In section 3, the new implementation of BEM is described. Numerical examples are given in Section 4. The paper ends with conclusions in Section 5 .

\section{The expanding element interpolation method}

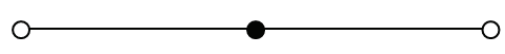

(a)

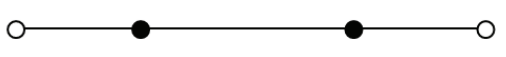

(b)

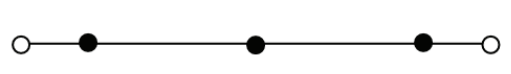

(c)

O Virtual nodes

- Collocation nodes

Fig. 1. (a) Constant expanding element; (b) Linear expanding element; (b) Quadratic expanding element.

In this section, the expanding element interpolation method is described in detail. As shown in Fig. 1, the collocation nodes and the virtual nodes constitute the higher order expanding element. For example, the shape functions of the constant expanding are 


$$
\left\{\begin{array}{l}
N_{v 1}=-0.5 \xi(1-\xi) \\
N_{v 2}=0.5 \xi(1+\xi) \\
N_{c}=(1-\xi)(1+\xi)
\end{array}\right.
$$

where $N_{v 1}, N_{v 2}$ and $N_{c}$ denote the shape functions of the virtual nodes and collocation node, respectively.

It can be seen that the interpolation accuracy of the proposed method increases by two orders compared with that of the original discontinuous element interpolation method. The values of virtual nodes are evaluated by different methods. Thus, the accuracy and convergence rate of the expanding element interpolation method are discussed in the following three cases.

2.1 The expanding elements used for interpolating the known boundary variables.

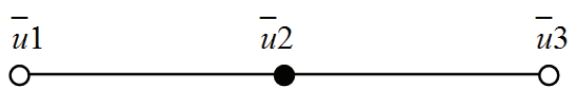

Fig. 2. The values of the virtual nodes equal the corresponding boundary conditions.

When interpolating the known boundary variables, the values of the virtual nodes equal the corresponding boundary conditions as shown in Fig. $2 . \bar{u} 1, \bar{u} 2$ and $\bar{u} 3$ in this figure represent the boundary conditions. It can be seen that the expanding elements become the continuous elements with the interpolation accuracy two orders higher than the original discontinuous elements. Therefore, the boundary conditions are accurately imposed.

2.2 The expanding elements used for interpolating the unknown boundary variables.

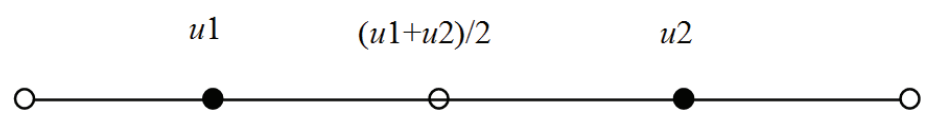

Fig. 3. The value of the virtual node between two constant expanding elements.

When interpolating the unknown boundary variables, the values of the virtual 
nodes are determined by all adjacent elements including the virtual nodes. The values of the virtual nodes are not independent variables. They depend on the values of the collocation nodes by the extrapolation of the original discontinuous elements. The extrapolation formulation is

$$
u^{v}=\frac{1}{2}\left(\sum_{i=1}^{n} N_{i}^{c} u_{i}^{c}+\sum_{j=1}^{m} N_{j}^{c} u_{j}^{c}\right)
$$

where $u^{v}$ represents the values of the virtual nodes. $N_{i}^{c}$ and $N_{j}^{c}$ are the shape functions of the adjacent discontinuous elements which include the virtual node, respectively. $u_{i}^{c}$ and $u_{j}^{c}$ denote the values of the collocation nodes.

For example, the value of the virtual node between two constant expanding elements equals the average value of these two elements as shown in Fig. 3. With this method, the interelement continuity can be guaranteed. To verify the accuracy and convergence, the expanding elements are used for interpolating the following function.

$$
f(x)=-x^{4}+3 x^{3}+x^{2}+1, \quad 0 \leq x \leq 4 \pi
$$

The relative errors and the convergence rates are shown in Fig. 4. In the following figures, $e$ denotes relative errors and $n$ stands for the number of the collocation nodes. Expdconst, ExpdLinear, ExpdQuad, TradLinear, TradQuad and TradCubic are the proposed expanding elements and traditional continuous elements interpolation methods with different orders, respectively. The relative errors $e$ is defined as

$$
e=\frac{1}{|u|_{\max }} \sqrt{\frac{1}{N} \sum_{i=1}^{N}\left(u_{i}^{r}-u_{i}^{s}\right)^{2}}
$$

where $|u|_{\max }$ is the maximum value over $N$ sample points; the superscripts $r$ and $s$ refer to the exact and numerical solutions, respectively. 


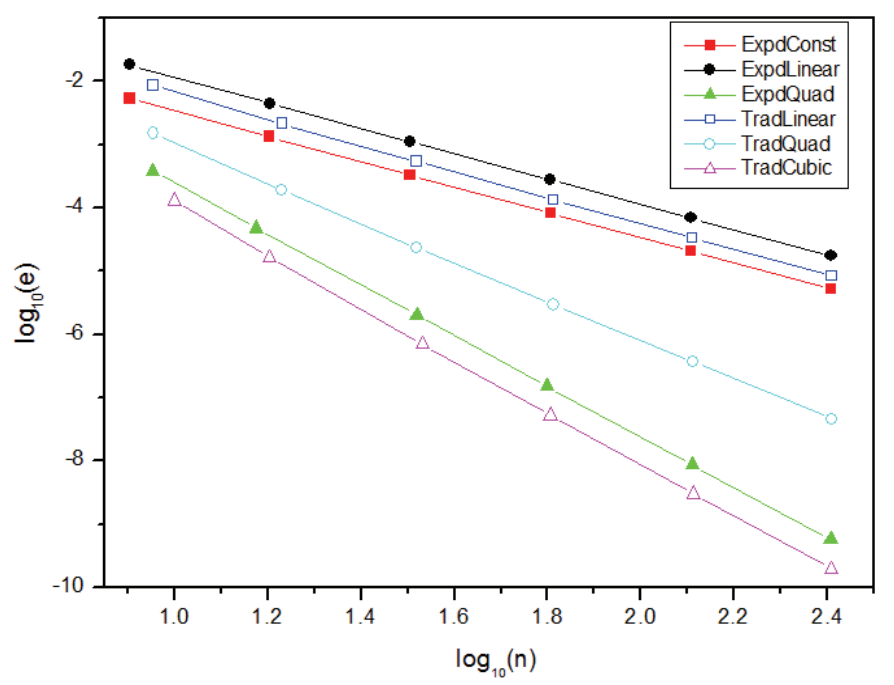

Fig. 4. The relative errors and the convergence rates for the expanding elements and traditional continuous elements.

It can be seen from Fig. 4 that the expanding elements possess almost the same convergence rates with the traditional continuous elements. But it is more convenient for the expanding elements to implement the BEM.

2.3 The expanding elements used for interpolating the discontinuous boundary variables

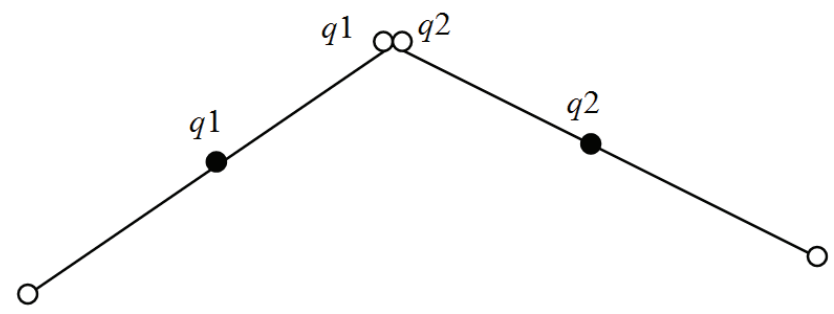

Fig. 5. The constant expanding elements for interpolating the discontinuous boundary variables.

When interpolating the discontinuous boundary variables, two virtual nodes are collocated at a vertex shared by two adjacent elements, one virtual node for one element, respectively. In order to accurately approximate the discontinuity of the boundary variable, the values of these two virtual nodes equal the values of corresponding elements, respectively, as shown in Fig. 5. To verify the accuracy and convergence, the following piecewise function with jump discontinuity are presented. 


$$
f(x)=\left\{\begin{array}{l}
(x+3)^{4},-2<x<0 \\
(x-3)^{4}+50,0 \leq x<2
\end{array}\right.
$$

The numerical results together with the exact solution are shown in Fig. 6. The relative errors and the convergence rates are shown in Fig. 7.

It can be seen from Fig. 6 and Fig. 7 that the discontinuity of boundary variables can be approximated accurately and the expanding element interpolation method has high rates of convergence.
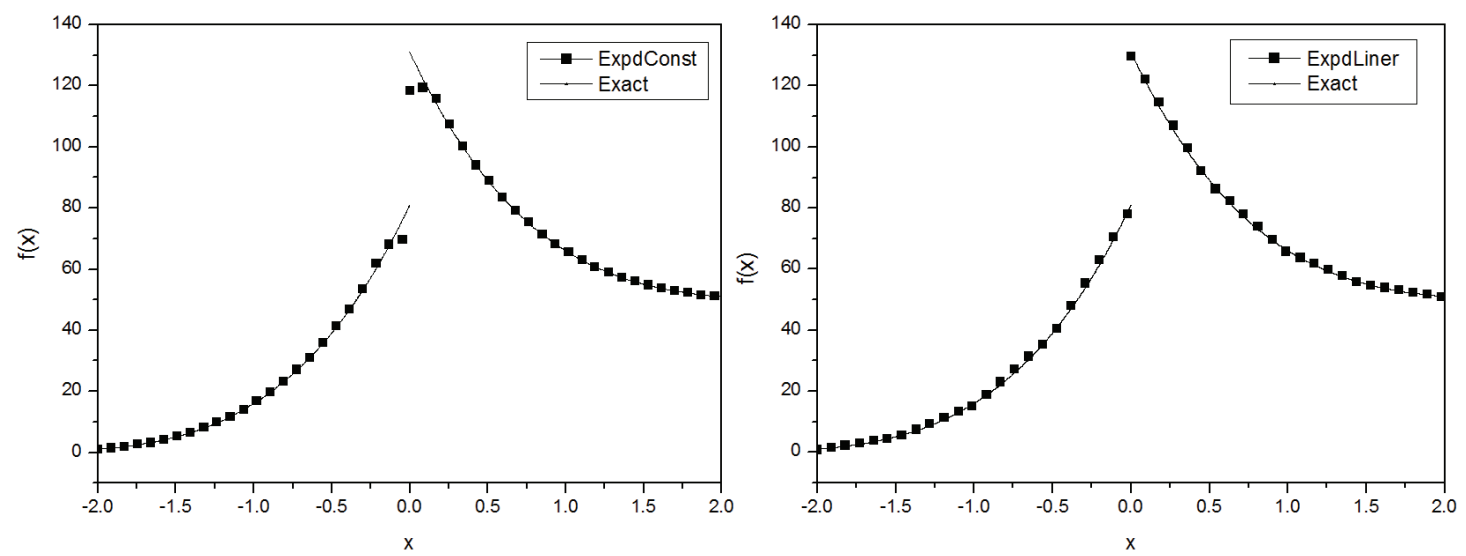

(a)

(b)

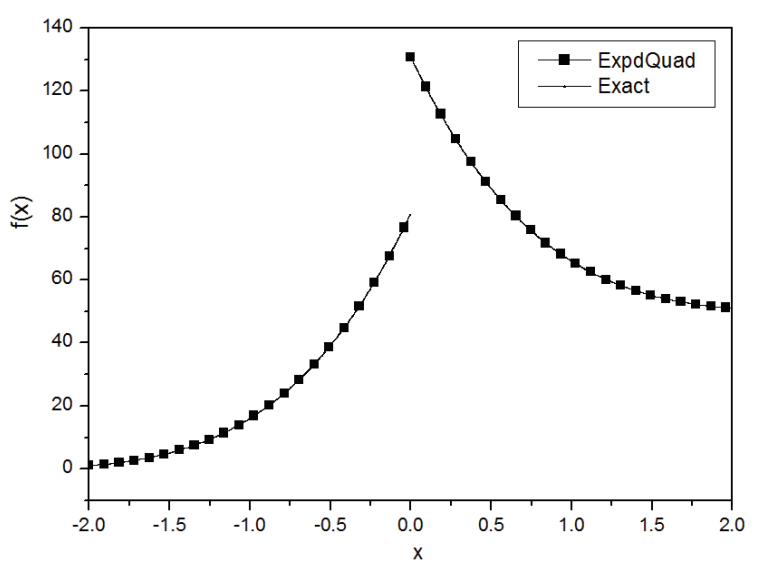

(c)

Fig. 6. The numerical results together with the exact solution (a) By constant expanding elements;

(b) By linear expanding elements; (c) By quadratic expanding elements. 


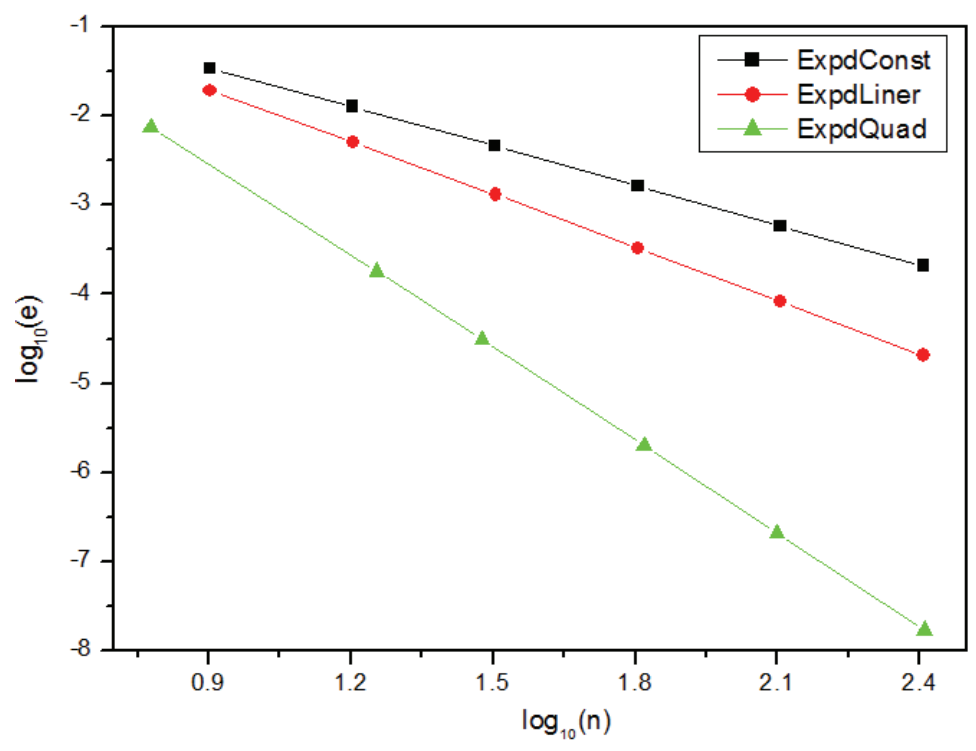

Fig. 7. The relative errors and the convergence rates for interpolating discontinuity

From the above analysis, it can be inferred that the expanding element interpolation method has high interpolation accuracy and convergence rates. The expanding elements not only guarantee the interelement continuity but also approximate the discontinuous boundary variables accurately.

\section{The new implementation of BEM by the expanding element interpolation method}

The well-known boundary integral equation (BIE) for the solution of Laplace's equation in a $2 \mathrm{D}$ region bounded by $\Gamma$ is

$$
c(\xi) u(\xi)+\int_{\Gamma} q^{*}(\xi, x) u(x) d \Gamma(x)=\int_{\Gamma} u^{*}(\xi, x) q(x) d \Gamma(x)
$$

where $\xi$ and $x$ are the source and the field points, respectively. $c(\xi)$ is a function of the internal angle of the boundary at point $\xi . u^{*}(\xi, x)$ is the fundamental solution to Laplace equation and

$$
q^{*}(\xi, x)=\frac{\partial u^{*}(\xi, x)}{\partial n(x)}
$$

Equation (6) is discretized with the $N$ expanding elements. The discretized equations for the BEM can be obtained.

$$
c(\xi) u^{j}(\xi)+\sum_{i=1}^{N}\left(\int_{\Gamma_{i}} q^{*}(\xi, x) \mathbf{N}^{T} d \Gamma(x)\right) \mathbf{r}^{i}=\sum_{i=1}^{N}\left(\int_{\Gamma_{i}} u^{*}(\xi, x) \mathbf{N}^{T} d \Gamma(x)\right) \mathbf{r}^{i}
$$


where $\mathbf{N}$ is a vector containing the shape functions of the expanding elements. $\mathbf{u}^{\mathrm{i}}$ and $\mathbf{q}^{\mathrm{i}}$ are the values of the collocation and virtual nodes in the $i^{\text {th }}$ expanding elements.

After the source point passes through all the collocation nodes, Equation (8) gives a system of linear equations, which can be expressed in a matrix form as

\section{$\mathbf{H u}=\mathbf{G t}$}

where vectors $\mathbf{u}$ and $\mathbf{t}$ consist of all nodal potential and normal flux. Matrix $\mathbf{H}$ contains integrals involving $q^{*}$, and matrix $\mathbf{G}$ contains integrals involving $u^{*}$.

$$
\begin{aligned}
& H_{i j}=\int_{\Gamma} q^{*}\left(\xi_{i}, x\right) N_{j} d \Gamma(x) \\
& G_{i j}=\int_{\Gamma} u^{*}\left(\xi_{i}, x\right) N_{j} d \Gamma(x)
\end{aligned}
$$

There are $\mathrm{n}$ collocation nodes and $\mathrm{m}$ virtual nodes. Distinguishing the known and unknown boundary variables, Equation (9) can be rewritten as

$$
\left[\begin{array}{llll}
\overline{\mathbf{H}}^{s} & \mathbf{H}^{s} & \overline{\mathbf{H}}^{v} & \mathbf{H}^{v}
\end{array}\right]\left[\begin{array}{l}
\overline{\mathbf{u}}^{s} \\
\mathbf{u}^{s} \\
\overline{\mathbf{u}}^{v} \\
\mathbf{u}^{v}
\end{array}\right]=\left[\begin{array}{llll}
\mathbf{G}^{s} & \overline{\mathbf{G}}^{s} & \mathbf{G}^{v} & \overline{\mathbf{G}}^{v}
\end{array}\right]\left[\begin{array}{l}
\mathbf{q}^{s} \\
\overline{\mathbf{q}}^{s} \\
\mathbf{q}^{v} \\
\overline{\mathbf{q}}^{v}
\end{array}\right]
$$

where $\overline{\mathbf{u}}^{s}, \overline{\mathbf{q}}^{s}$ and $\mathbf{u}^{s}, \mathbf{q}^{s}$ are the known and unknown boundary variables of the collocation nodes, respectively. $\overline{\mathbf{u}}^{v}, \overline{\mathbf{q}}^{v}$ and $\mathbf{u}^{v}, \mathbf{q}^{v}$ represent the known and unknown boundary variables of the virtual nodes, respectively.

$$
\begin{gathered}
\overline{\mathbf{u}}^{s}=\left[\begin{array}{c}
\bar{u}_{1}^{s} \\
\bar{u}_{2}^{s} \\
\mathbf{?} \\
\bar{u}_{i}^{s}
\end{array}\right], \mathbf{u}^{s}=\left[\begin{array}{c}
u_{i+1}^{s} \\
u_{i+2}^{s} \\
u_{n}^{s}
\end{array}\right], \overline{\mathbf{u}}^{v}=\left[\begin{array}{c}
\bar{u}_{n+1}^{v} \\
\bar{u}_{n+2}^{v} \\
\mathbf{?} \\
\bar{u}_{j}^{v}
\end{array}\right], \mathbf{u}^{v}=\left[\begin{array}{c}
u_{j+1}^{v} \\
u_{j+2}^{v} \\
\\
u_{n+m}^{v}
\end{array}\right] \\
\mathbf{q}^{s}=\left[\begin{array}{c}
q_{1}^{s} \\
q_{2}^{s} \\
\mathbf{?} \\
q_{i}^{s}
\end{array}\right], \overline{\mathbf{q}}^{s}=\left[\begin{array}{c}
\bar{q}_{i+1}^{s} \\
\bar{q}_{i+2}^{s} \\
\bar{q}_{n}^{s}
\end{array}\right], \mathbf{q}^{v}=\left[\begin{array}{c}
q_{n+1}^{v} \\
q_{n+2}^{v} \\
\mathbf{?} \\
q_{j}^{v}
\end{array}\right], \overline{\mathbf{q}}^{v}=\left[\begin{array}{c}
\bar{q}_{j+1}^{v} \\
\bar{q}_{j+2}^{v} \\
\bar{q}_{n+m}^{v}
\end{array}\right]
\end{gathered}
$$

And $\overline{\mathbf{H}}^{s}, \mathbf{H}^{s}, \overline{\mathbf{H}}^{v}, \mathbf{H}^{v}, \mathbf{G}^{s}, \overline{\mathbf{G}}^{s}, \mathbf{G}^{v}, \overline{\mathbf{G}}^{v}$ are given by

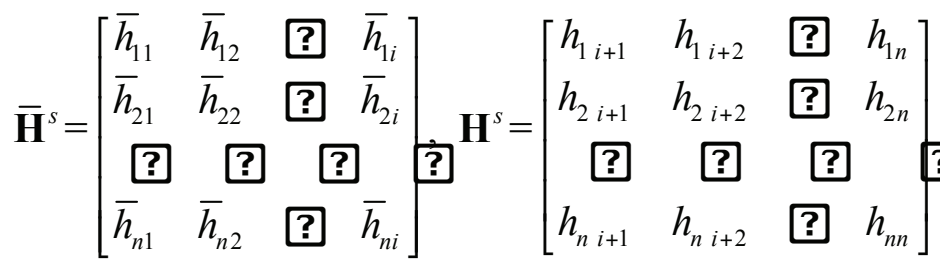




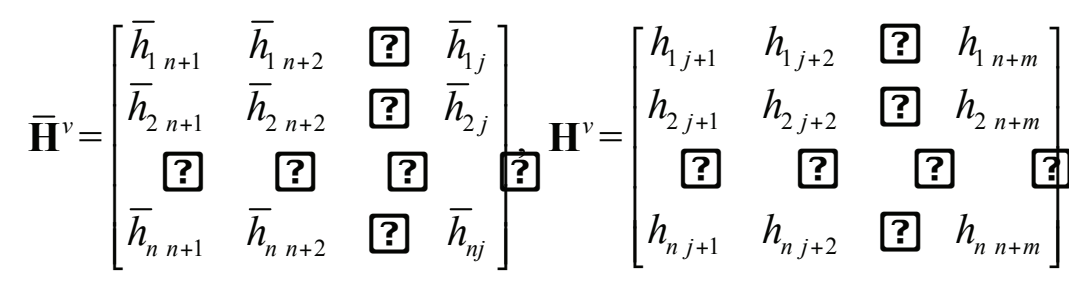

$$
\begin{aligned}
& \mathbf{G}^{s}=\left[\begin{array}{cccc}
g_{11} & g_{12} & \text { ? } & g_{1 i} \\
g_{21} & g_{22} & \text { ? } & g_{2 i} \\
? & \text { ? } & \text { ? } \\
g_{n 1} & g_{n 2} & \text { ? ? } & g_{n i}
\end{array}\right], \overline{\mathbf{G}}^{s}=\left[\begin{array}{ccc}
\bar{g}_{1 i+1} & \bar{g}_{1 i+2} & \text { ? } \bar{g}_{1 n} \\
\bar{g}_{2 i+1} & \bar{g}_{2 i+2} & \text { ? } \bar{g}_{2 n} \\
? & ? & ? \\
\bar{g}_{n i+1} & \bar{g}_{n i+2} & \text { ? } \bar{g}_{n n}
\end{array}\right]
\end{aligned}
$$

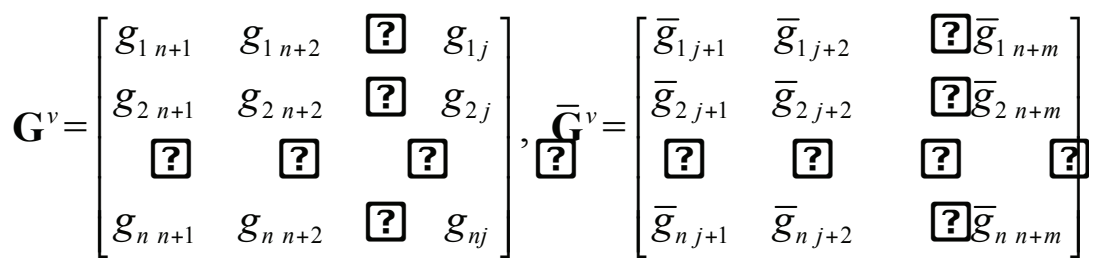

From Equations (14)-(17), one can see that the matrices $\mathbf{H}$ and $\mathbf{G}$ are no longer square matrix since the virtual nodes are not used for the source points. But from Equation (2) in Section 2, we can know that $\mathbf{u}^{v}$ and $\mathbf{q}^{v}$ are not truly independent variables. They depend on the values of the corresponding collocation nodes.

$$
\begin{aligned}
\mathbf{u}^{v} & =\mathbf{N}_{1} \mathbf{u}^{s} \\
\mathbf{q}^{v} & =\mathbf{N}_{2} \mathbf{q}^{s}
\end{aligned}
$$

where $\mathbf{N}_{1}$ and $\mathbf{N}_{2}$ contain the shape functions of the original discontinuous elements, which are computed by the methods descript in Section 2. Substituting Equation (18) into Equation (11) and rearranging Equation (11) according to the boundary conditions, the final system of linear equations can be obtained.

$$
\mathbf{A x}=\mathbf{f}
$$

where

$$
\begin{gathered}
\mathbf{A}=\left[\begin{array}{ll}
\mathbf{H}^{s}+\mathbf{H}^{v} \mathbf{N}_{1} & -\left(\mathbf{G}^{s}+\mathbf{G}^{v} \mathbf{N}_{2}\right)
\end{array}\right] \\
\mathbf{x}=\left[\begin{array}{l}
\mathbf{u}^{s} \\
\mathbf{q}^{s}
\end{array}\right] \\
\mathbf{f}=\left[\overline{\mathbf{G}}^{s} \overline{\mathbf{q}}^{s}+\overline{\mathbf{G}}^{v} \overline{\mathbf{q}}^{v}-\overline{\mathbf{H}}^{s} \overline{\mathbf{u}}^{s}-\overline{\mathbf{H}}^{v} \overline{\mathbf{u}}^{v}\right]
\end{gathered}
$$

Matrix A matrix is a square matrix of order $n . \mathbf{x}$ is the vector containing $n$ boundary unknowns at the source points, only. $\mathbf{f}$ is the known vector on the right-hand side.

It is interesting to note that, from Equation (19)-(22), the size of the overall system 
of linear equations is just the same as that in the traditional discontinuous element implementation. The variables at the virtual nodes do not appear in the overall system of equations. This point is the main ingredient of the merit of our method.

\section{Numerical examples}

To verify the accuracy and efficiency of the proposed method, several examples with different boundary conditions are presented in this section. In all cases, the Laplace equation

$$
\nabla^{2} u=0
$$

is solved. For the purpose of error estimation and convergence studies, Equation (4) is used. In all figures, $e$ denotes relative errors and $n$ stands for the number of the collocation nodes. Expdconst, ExpdLinear, ExpdQuad, TradLinear, TradQuad and TradCubic are the proposed expanding elements and traditional continuous elements interpolation methods with different orders, respectively.

\subsection{Example 1}

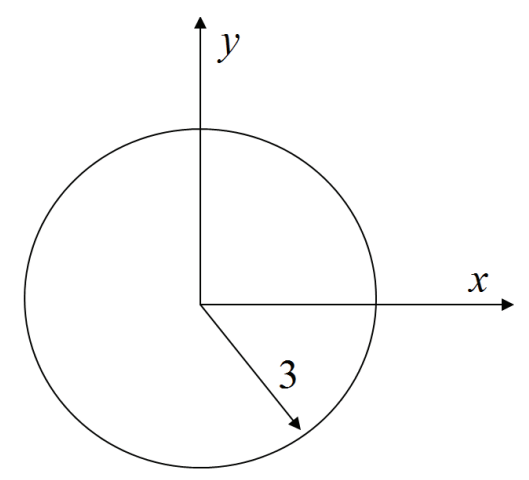

Fig. 8. Dirichlet problem on a circle

A Dirichlet problem on a circle of radius 3 unit, centered at the origin (see Fig. 8) is concerned in this example. The exact solution is

$$
u=-x^{3}-y^{3}+3 x^{2} y+3 x y^{2}
$$

The essential boundary condition is imposed on the whole circle. Numerical results of $u$ along the radius (from $(0,0)$ to $(3,0)$ ) from the expanding elements and traditional continues elements interpolation method, together with the exact solution, 
are shown in Fig. 9. To study the convergence of the proposed method, different number of collocation node is used for $u$ as shown in Fig. 10.

From Fig. 9 and Fig. 10, it can be seen that the results for $u$ by the proposed method are accurate, and high convergence rates can be obtained.

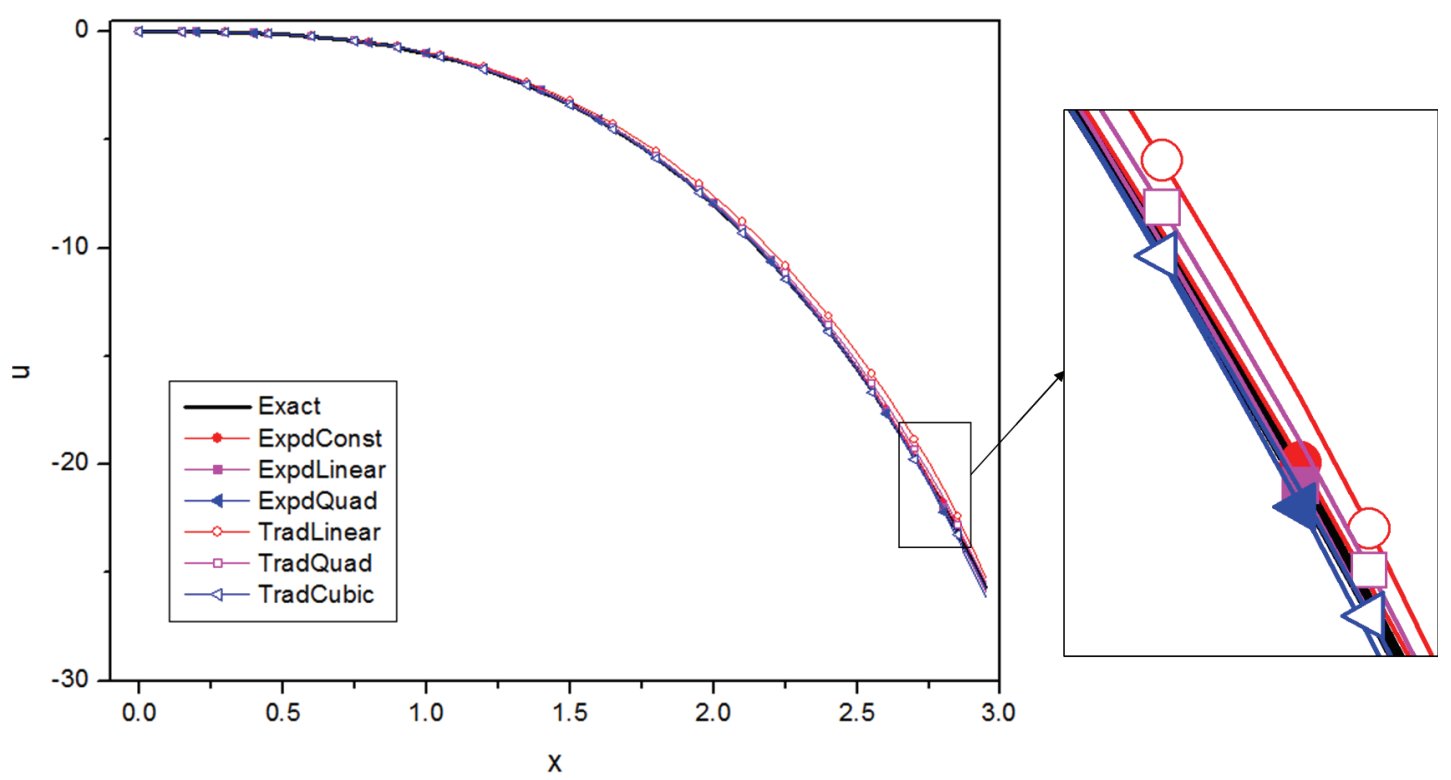

Fig. 9. $u$ along the radius (from $(0,0)$ to $(3,0)$ ).

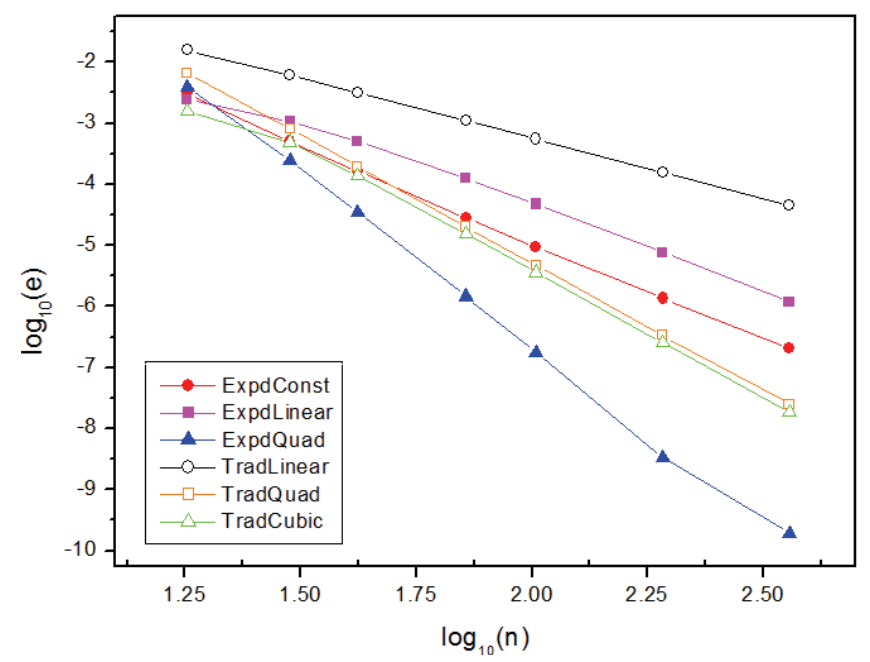

Fig. 10. The relative errors and convergence rates of $u$ along the radius (from $(0,0)$ to $(3,0)$ ).

\subsection{Example 2}




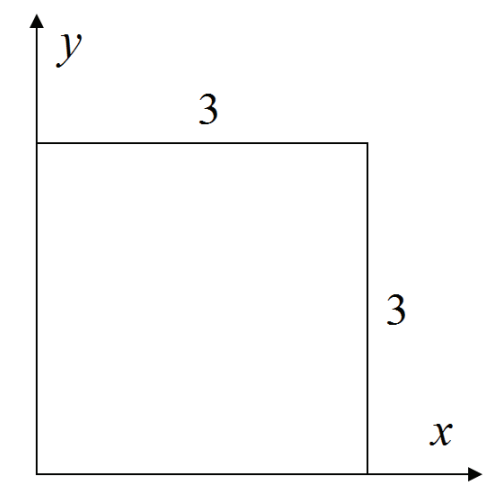

Fig. 11. Mixed problem on a square

A mixed problem on a $3 \times 3$ square as shown in Fig. 11 is presented in the second example. The exact solution for this problem is given by

$$
u=-x^{4}-y^{4}+6 x^{2} y^{2}
$$

The essential boundary condition is imposed on the left and top edges and the flux boundary condition is prescribed on the right and bottom edges of the domain. The flux boundary condition has a jump discontinuity at the point $(3,0)$. The numerical results of $u$ on the diagonal (from $(0,0)$ to $(3,3)$ ), together with the exact solution are shown in Fig. 12. The relative errors and convergence of $u$ on the right and bottom edges are shown in Fig. 13.

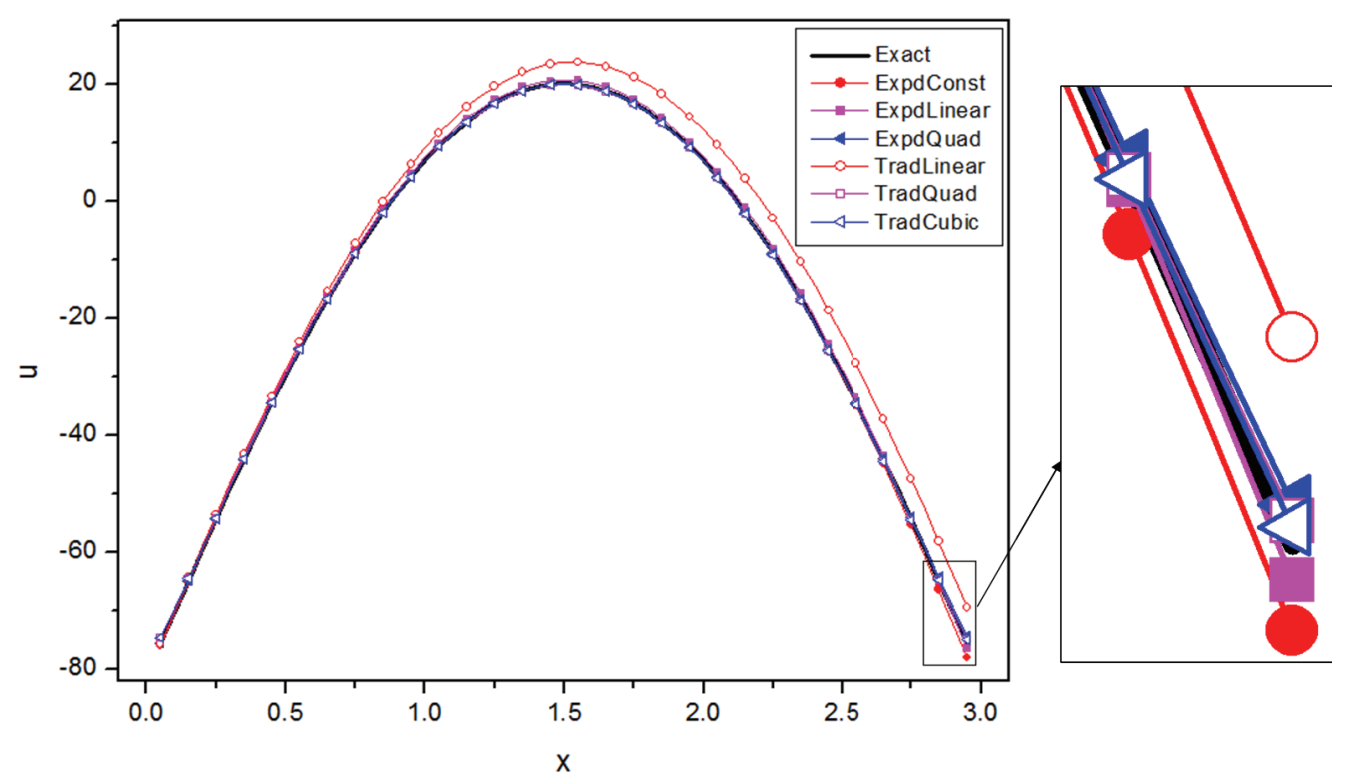

Fig. 12. $u$ along the diagonal (from $(0,0)$ to $(3,3)$ ). 


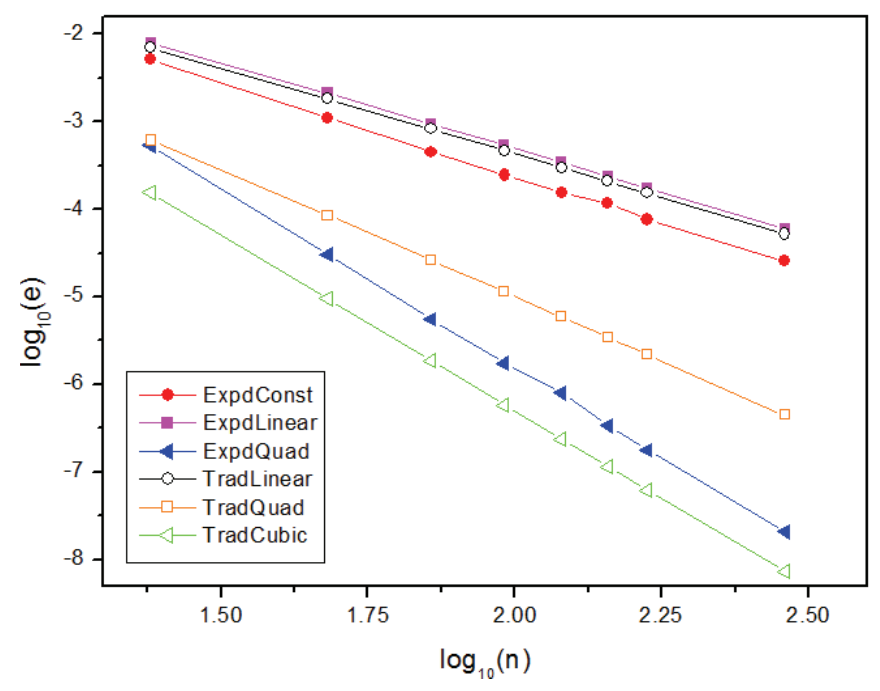

Fig. 13. The relative errors and convergence rates of $u$ on the right and bottom edges of the square

As illustrated in this example that the discontinuity of $q$ can be accurately approximated and the agreement between numerical and exact results of $u$ are excellent. Thus, continuous and discontinuous fields on the boundary can be accurately approximated by the proposed method.

\subsection{Example 3}

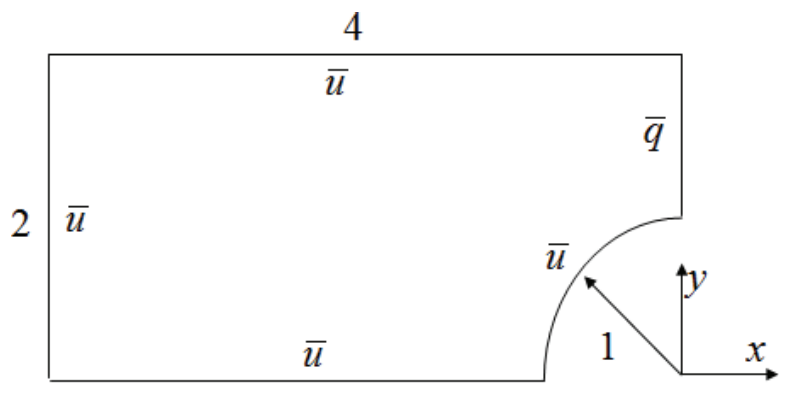

Fig. 14. Mixed problem with complex geometry

A mixed problem with complex geometry is considered in the last example as shown in Fig. 14. The exact solution is

$$
u=y\left[1-\frac{1}{x^{2}+y^{2}}\right]
$$

The prescribed $u$ and $q$ values along all boundaries are shown in Fig. 14. The numerical results of $u$ on the diagonal (from $(-4,0)$ to $(0,2)$ ), together with the exact 
solution are shown in Fig. 15. The relative errors and convergence of $q$ along the boundaries where the essential boundary condition is imposed are shown in Fig. 16.

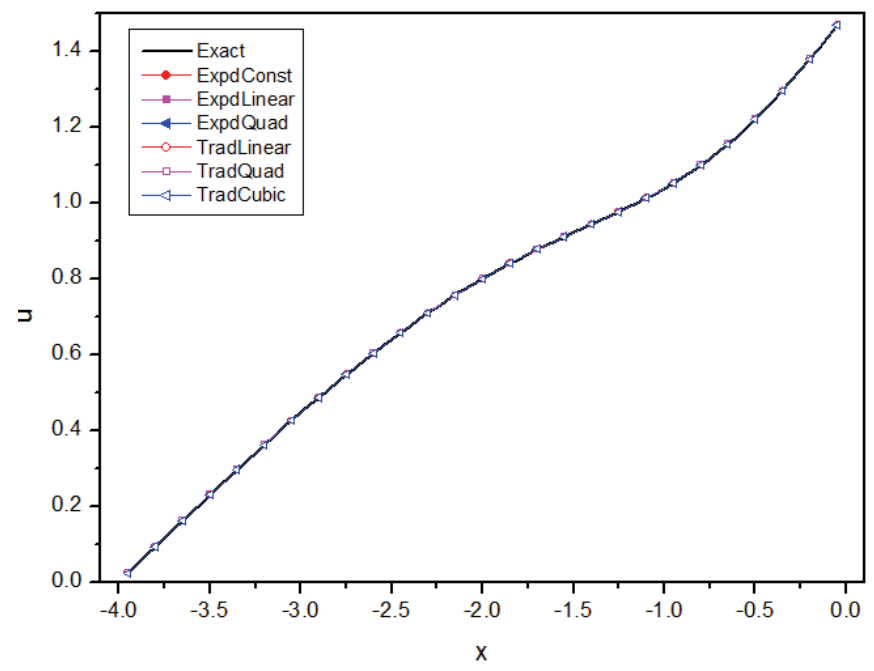

Fig. 15. $u$ along the diagonal (from $(-4,0)$ to $(0,2)$ ).

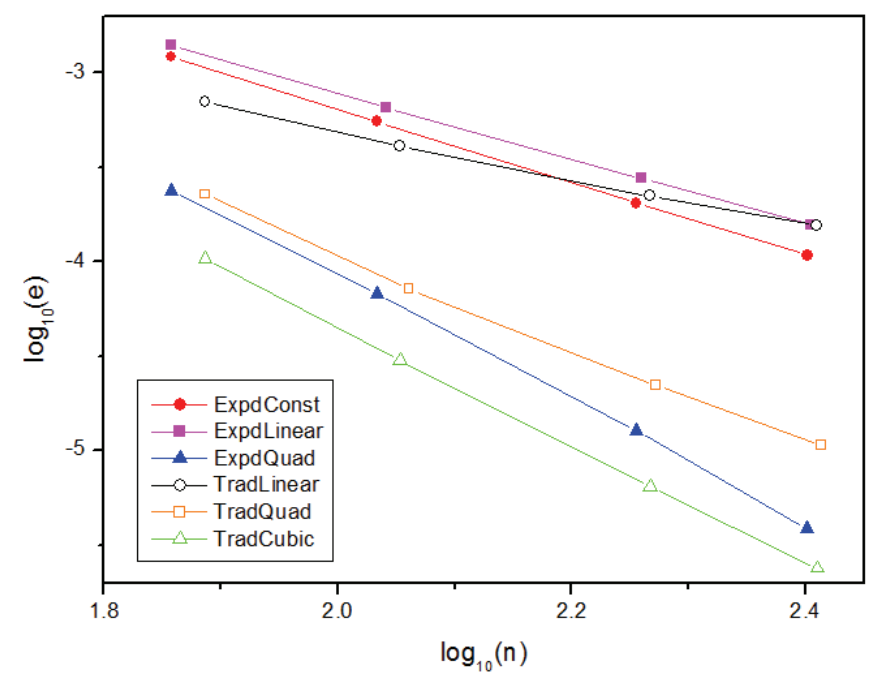

Fig. 16. The relative errors and convergence rates of $q$.

In Fig. 15 and Fig. 16, it can be noted that for the complex geometry, the proposed method possess high accuracy and convergence rate again. This shows that the expanding element interpolation method is feasible. Furthermore, the implementation of BEM by the expanding element is easier than that by the traditional continuous elements.

\section{Conclusions}


A new implementation of BEM by the expanding element interpolation method is proposed in this paper. The interpolation accuracy and efficiency of the proposed elements is higher than that of the traditional discontinuous elements. The expanding element inherits the advantages of the discontinuous element, thus the implementation of BEM by the expanding element is more convenient than that by the traditional continuous element. Both continuous and discontinuous fields on the boundary can be accurately approximated by the expanding elements. The numerical examples showed that the proposed method possesses satisfactory accuracy and convergence rate compared with the traditional continuous element interpolation method. Through different collocation of the virtual nodes, the proposed method can be applied to solve the discontinuous problems such as contact problems and crack propagation, which is planned in near future.

\section{Acknowledgements}

This work was supported in part by National Science Foundation of China under grant number 11472102, in part by the Collaborative Innovation Center of Major Machine Manufacturing in Liaoning.

\section{References}

1. Brebbia CA, Telles JCF, Wrobel LC. Boundary element techniques: theory and applications in engineering. Vol. 5. Berlin: Springer-Verlag; 1984.

2. Cheng AHD, Cheng DT. Heritage and early history of the boundary element method. Engineering Analysis with Boundary Elements 2005; 29; 268-302.

3. Yao ZH. A new type of high-accuracy BEM and local stress analysis of real beam, plate and shell structures. Engineering Analysis with Boundary Elements 2016; 65; 1-17.

4. Zhang JM, Qin XY, Han X, Li GY. A boundary face method for potential problems in three dimensions. International Journal for Numerical Methods in Engineering 2009; 80; 320-337.

5. Zhou FL, Zhang JM, Sheng XM, Li GY. A dual reciprocity boundary face method for 3D non-homogeneous elasticity problems. Engineering Analysis with Boundary Elements 2012; 36; 1301-1310.

6. Zhuang C, Zhang JM, Qin XY, Zhou FL, Li GY. Integration of subdivision method into boundary element analysis. International Journal of Computational Methods 2012; 09; 367-374. 
7. Xie GZ, Zhang JM, Huang C, Lu CJ, Li GY. A direct traction boundary integral equation method for three-dimension crack problems in infinite and finite domains. Computational Mechanics 2014; 53; 575-586.

8. Zhang JM, Huang C, Lu CJ, Han L, Wang P, Li GY. Automatic thermal analysis of gravity dams with fast boundary face method. Engineering Analysis with Boundary Elements 2014; $41 ; 111-121$.

9. Dong YQ, Zhang JM, Xie GZ, Lu CJ, Han L, Wang P. A general algorithm for the numerical evaluation of domain integrals in 3D boundary element method for transient heat conduction. Engineering Analysis with Boundary Elements 2015; 51; 30-36.

10. Chen JT, Hong HK. Review of dual boundary element methods with emphasis on hypersingular integrals and divergent series. Applied Mechanics Reviews 1999; 52; 17-33.

11. Liu YJ. Analysis of shell-like structures by the boundary element method based on 3-D elasticity: formulation and verification. International Journal for Numerical Methods in Engineering 1998; 41; 541-558.

12. Johnston BM, Johnston PR, Elliott D. A new method for the numerical evaluation of nearly singular integrals on triangular elements in the 3D boundary element method. Journal of Computational and Applied Mathematics 2013; 245; 148-161.

13. Maestre J, Cuesta I, Pallares J. An unsteady 3D Isogeometrical Boundary Element Analysis applied to nonlinear gravity waves. Computer Methods in Applied Mechanics and Engineering 2016; Accepted.

14. Rosen D, Cormack DE. On corner analysis in the BEM by the continuation approach. Engineering Analysis with Boundary Elements 1995; 16; 53-63.

15. Shen J, Sterz O. A mixed galerkin and collocation approach for treating edge and corner problems in the boundary element method. Magnetics IEEE Transactions on 1998; 34; 3296-3299.

16. Mukhopadhyay S, Majumdar N. A study of three-dimensional edge and corner problems using the neBEM solver. Engineering Analysis with Boundary Elements 2009; 33; 105-119.

17. Guiggiani M, Krishnasamy G, Rudolphi TJ, Rizzo FJ. A general algorithm for the numerical solution of hypersingular boundary integral equations. Journal of Applied Mechanics 1992; $59 ; 604-614$.

18. Cisilino A. Linear and nonlinear crack growth using boundary elements. Southampton: WIT Press; 2000.

19. Manolis GD, Banerjee PK. Conforming versus non-conforming boundary elements in threedimensional elastostatics. International Journal for Numerical Methods in Engineering 1986; $23 ; 1885-1904$.

20. Parreira P. On the accuracy of continuous and discontinuous boundary elements. Engineering Analysis 1988; 5; 205-211. 